\title{
Treatment of eating disorders in child and adolescent psychiatry
}

\author{
Beate Herpertz-Dahlmann
}

\begin{abstract}
Purpose of review
Recent research on the multimodal treatment of eating disorders in child and adolescent psychiatry has yielded a significant increase in randomized controlled trials and systematic reviews. This review aims to present relevant findings published during the last 2 years related to medical and psychological treatment of anorexia nervosa, bulimia nervosa and avoidant/restrictive food intake disorder (ARFID).
\end{abstract}

\section{Recent findings}

For anorexia nervosa, recent reports described the efficacy of different treatment settings, lengths of hospital stay and high vs. low-calorie refeeding programmes. For both anorexia and bulimia nervosa, a number of randomized controlled trials comparing individual and family-oriented treatment approaches were published. For the newly defined ARFID, only very preliminary results on possible treatment approaches implying a multidisciplinary treatment programme were obtained.

\section{Summary \\ Although there is some evidence of the effectiveness of new child and adolescent psychiatric treatment approaches to eating disorders, the relapse rate remains very high, and there is an urgent need for ongoing intensive research.}

\section{Keywords}

anorexia nervosa, avoidant/restrictive eating disorder, bulimia nervosa, child and adolescent psychiatry, eating disorders, treatment

\section{INTRODUCTION}

In adolescence and young adulthood, the disabilityadjusted life years (DALYs) of eating disorders are one of the highest among all mental disorders. In the Global Burden of Disease Study 2013, anorexia nervosa and bulimia nervosa combined were the 12th leading cause of DALYs in 15-19-year-old girls in high-income countries, accounting for $2.2 \%$ of all DALYs $[1,2=3]$. However, these statistical numbers do not reflect the overall economic and emotional burden to society and families, although in this age group, caregivers are still of significant relevance [ $\left.2^{-}\right]$.

Anorexia nervosa and atypical anorexia nervosa are by far the most prevalent eating disorders among patients admitted to child and adolescent psychiatric (CAP) treatment or to paediatric units with a CAP liaison service, whereas patients with bulimia nervosa and avoidant/restrictive food intake disorder (ARFID) are seen comparatively less often. Individuals with binge eating disorder (BED) are mostly seen in specialist services for obesity. Thus, this article will specifically address the medical and psychological treatment of young individuals with threshold and subthreshold forms of anorexia nervosa. Recent years have evidenced a notable increase in large and methodologically sound studies. Several important randomized controlled trials (RCTs) and systematic reviews comparing different treatment settings, refeeding practices and psychotherapeutic interventions for adolescent and childhood anorexia nervosa have been recently published. This article will introduce an important research development to provide an evidence-based approach to current treatment methods and practices for young patients.

Department of Child \& Adolescent Psychiatry, Psychosomatics and Psychotherapy, University Clinics, Technical University Aachen, Aachen, Germany

Correspondence to Beate Herpertz-Dahlmann, Department of Child \& Adolescent Psychiatry, Psychosomatics and Psychotherapy, University Clinics, Technical University Aachen, Neuenhofer Weg 21, D-52074 Aachen, Germany. Tel: +49 241 8088737; e-mail: bherpertz@ukaachen.de

Curr Opin Psychiatry 2017, 30:438-445

DOI:10.1097/YCO.0000000000000357

This is an open access article distributed under the terms of the Creative Commons Attribution-Non Commercial-No Derivatives License 4.0 (CCBY-NC-ND), where it is permissible to download and share the work provided it is properly cited. The work cannot be changed in any way or used commercially without permission from the journal. 


\section{KEY POINTS}

- There is emerging evidence that less-intensive treatment settings, such as day patient or outpatient treatment approaches, achieve similar outcomes as inpatient/ residential treatment in medically stable adolescent patients.

- A multidisciplinary team experienced in the treatment of eating disorders delivering a multimodal treatment is most appropriate to support the patient and her caregivers in overcoming the eating disorder.

- Higher caloric refeeding at the beginning of nutritional rehabilitation in patients with anorexia nervosa is not associated with an increased risk for medical complications but is associated with improved weight gain.

- Many ways lead to Rome: although family-based therapy achieves a symptom remission in a shorter period of time for anorexia and bulimia nervosa, individual interventions do not seem to be less effective in the long run. Parent-focused treatment with the patient treated separately does not seem to be inferior to conjoint family-based therapy.

- For ARFID, very preliminary results indicate the benefit of a multidisciplinary treatment team, behavioural intervention as a central treatment element and involvement of the caregiver.

However, up-to-date research on CAP treatment of childhood and adolescent bulimia nervosa and ARFID will also be presented.

\section{ANOREXIA NERVOSA}

In a recent nationwide Danish study, the incidence of anorexia nervosa doubled between 1995 and 2010 from 10 to 19/100 000 persons/year, whereas the age of onset decreased during the observation period [4]. Several European surveys indicate a significant increase in rates of hospital admission for childhood and adolescent anorexia nervosa. In Germany, the number of individuals under 15-year olds treated in hospitals rose from six to $15 / 100000$ persons between 2005 and 2014 (https://www.gbe-bund.de). Similarly, in the United Kingdom, admission rates in the adolescent age group rose from six to nearly 16/100 000 persons during the last 20 years. Among other factors, changing diagnostic practices and a better knowledge of eating disorders might explain these increasing rates $\left[5^{-}\right]$.

\section{TREATMENT SETTINGS}

The mortality risk, with a standardized mortality rate of 11.5 by 1 year after discharge, in individuals with anorexia nervosa aged 15-24 years is higher than that for other serious diseases in adolescence, such as asthma, type 1 diabetes and any other psychiatric disorder [6]. Nonetheless, differences in national healthcare politics lead to differences in populations treated in inpatient CAP or paediatric units. In most countries, special healthcare services for eating disorders are rare. In the United Kingdom, several specialist eating disorder national health services have been established recently, resulting in a significantly shortened duration of time until the patient has contact with the specialist service and duration of eating disorder before treatment [7"]. In a recent study by Schmidt and coworkers, significantly more patients treated faster at the specialist service had normalized their BMI after 1 year than those treated under usual conditions (TAU) (McClelland et al., unpublished data). However, the sample comprised only young adults and did not include adolescents.

\section{Inpatient treatment and length of hospital stay}

In central Europe, most adolescents with anorexia nervosa are still treated in hospitals, whereas many patients in Anglo-Saxon countries are seen only as outpatients. However, there are some medical indications for hospital admission that have been widely agreed upon (Table 1).

If inpatient treatment is necessary, most clinical guidelines recommend a CAP or paediatric unit with a 'developmentally aware and sensitive staff' experienced in the treatment of eating disorders $[8,9]$. A multimodal approach with an interdisciplinary team working closely together is the treatment of choice (Table 2). Depending on the healthcare system, child and adolescent psychiatrists and/or paediatricians, dieticians, physiotherapists and

Table 1. Medical and psychosocial indications for hospital admission for adolescent anorexia nervosa

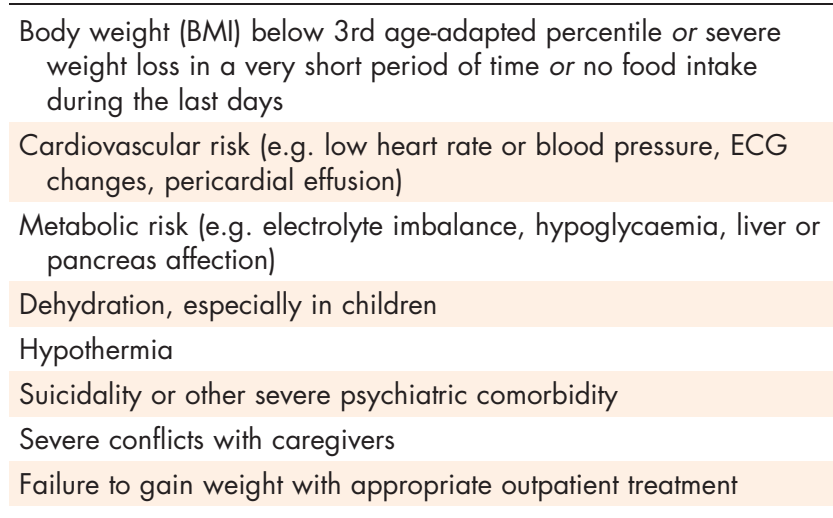

Summary Clinical Guidelines [8,9]; German S3-guidelines 2nd edition (Herpertz, Herpertz-Dahlmann, et al. unpublished). 
Table 2. Multimodal child and adolescent psychiatric treatment of adolescent and childhood anorexia nervosa

Nutritional counselling

Nutritional rehabilitation

Treatment of medical and psychiatric comorbidity

Individual and group psychotherapeutic interventions

Family-oriented treatment strategies

experienced nurses compose the core professionals. This applies for all treatment settings.

To compare the effectiveness of a prolonged hospitalization for weight restoration with that of a shorter stay for medical stabilization, Madden et al. [10] conducted a RCT with Australian adolescent inpatients. After discharge, all patients received 20 additional sessions of outpatient family-based therapy (FBT). At the 12-month follow-up, there was no significant difference in hospital bed use between the two groups with either short (22 days) or longer hospitalization times (37 days) at baseline. However, healthcare costs were significantly lower in the 'only' medically stabilized group. This result is interesting because it confirms doubts about the effect of inpatient treatment. Note, however, that the results of this study might be biased by cluster randomization.

\section{Day patient treatment}

After medical stabilization, there might be some major advantages of day patient in comparison with inpatient treatment for adolescents: new skills obtained in treatment might be more easily transferred home; the parents and family exhibit greater involvement; the patient may remain in her/his social networks and develop more age-adequate autonomy.

In our own multicentre stepped care trial, 172 adolescents with first onset anorexia nervosa were included. After 3 weeks of inpatient treatment, patients were randomly assigned to either inpatient treatment or day patient treatment. After 1 year, day patient was not inferior to inpatient treatment: BMI was similar in the two groups, mental state (especially depression) and psychosexual development were significantly better in the day patient group, and healthcare costs were significantly lower [11"]. Moreover, after an observation period of 2.5 years, the day patient group exhibited a higher BMI and significantly fewer relapses and readmissions to hospital (Herpertz-Dahlmann, unpublished data).

\section{Comparison of different treatment settings}

A systematic review by Madden [12] evaluated five studies, including three studies with adolescents.
Two of the studies compared inpatient with outpatient treatment, one compared inpatient with day patient treatment [11"], one compared day patient with outpatient treatment and one compared different lengths of inpatient treatment [10]. The authors found no major difference in outcome defined by BMI and changes in eating disorder psychopathology between treatment settings and lengths of treatment. However, inpatient treatment was significantly more expensive than all other treatment settings. In another narrative review of the outcome of 22 trials investigating partial and residential treatment of adult and adolescent anorexia nervosa, a similar result was obtained. Most studies showed an improvement in the disorder at discharge that was sustained for a length of time after discharge. Nevertheless, long-term efficiency, especially in residential/inpatient treatment, is yet to be demonstrated [13"].

Note, however, that studies on different treatment settings are difficult to compare if they are not conducted as RCTs. For example, in the US trials investigating FBT in adolescent outpatients, participants usually have a higher BMI at admission in comparison with those in European clinical studies, and many patients had been hospitalized directly before or during treatment [14]; for a review see [15].

\section{NUTRITIONAL REHABILITATION}

In the treatment of adolescent anorexia nervosa, restoration of a healthy body weight implying age-appropriate growth and resumption of menses is one of the major goals. However, there is no consensus about the definition of a healthy body weight and, consequently, of a target weight. Recent studies on adolescent anorexia nervosa indicate an age-adapted BMI percentile of 25 as a necessary condition for the resumption of menses $[16,17]$; thus, this value may be recommended as a reference weight for the majority of adolescent patients.

There is an ongoing discussion about how to proceed in refeeding severely malnourished patients. According to a systematic review with children and adolescents and corresponding to usual clinical practice, the initially prescribed energy intake varies widely between 1000 and more than $1900 \mathrm{kcal}$ [18]. Many clinicians are afraid of the socalled refeeding syndrome with severe cardiac, renal and neurological complications. However, a recent observational study [19], a systematic review of 27 studies with adults and adolescents [20"] and a small RCT [21"'], did not find any difference in the occurrence of the refeeding syndrome in adolescents started on a lower vs. a higher caloric diet. 
The systematic review came to the following conclusions: lower caloric refeeding is too conservative for mildly and moderately starved patients; both meal-based refeeding approaches and combined nastrogastric tube feeding and meals can provide a high supply of energy; higher calorie refeeding has not been associated with increased risk for the refeeding syndrome under close medical observation, including electrolyte balancing; there is insufficient evidence to change the current clinical practice for severely emaciated patients, for example administering an initially low supply of energy; parenteral nutrition is not recommended; components of nutrition (e.g. protein, carbohydrates and fat) should be within recommended ranges; more research in nonhospital settings is needed; there is insufficient knowledge on the long-term outcomes of different approaches [20"]. In the small RCT mentioned above, 18 patients aged 10-16 years were randomized to a diet starting at either $500 \mathrm{kcal} /$ day (TAU) or $1200 \mathrm{kcal} /$ day. The latter diet was associated with a higher BMI after 10 days but not with a higher rate of medical problems, including hypophosphatemia [21"']. Rather, hypophosphatemia occurred in patients with a low BMI and reduced white blood cells at intake.

To re-establish normal eating behaviours, an individualized meal plan consisting of three main meals and three snacks is recommended. In an outpatient setting, caregivers should be given help to support their child during the meal through a family meal, group psychoeducation for parents or personal instruction from the therapist $[22,23]$.

\section{MEDICATION}

To date, no medication for adolescent anorexia nervosa has been approved by the US Food and Drug Administration or the European Medicines Agency. Furthermore, empirical evidence demonstrates the ineffectiveness of medication for this eating disorder. However, the use of medication, particularly olanzapine, might be necessary on an intermittent basis for highly anxious and agitated patients (for more information, refer to [15]).

\section{PSYCHOLOGICAL APPROACHES}

Psychotherapy is an essential part of CAP treatment of anorexia nervosa delivered on an individual basis or family-oriented approach in inpatient, day patient or outpatient treatment.

Because of the considerable acceptance of Cognitive-Behavioral Therapy (CBT) for nonrestrictive eating disorders [24], this treatment approach has also been designed to treat all forms of eating disorders, including anorexia nervosa, from outpatient to inpatient settings (CBT enhanced, CBT-E). Based primarily on Fairburn's [25] concept for adults, CBT-E in adolescents also includes regular sessions for caregivers. Data from two recent Italian studies of CBT-E indicate that it is effective for both adolescent inpatient and outpatient $[26,27]$. However, there was no control group in either study.

Another individual psychological treatment mainly practiced in the United States is adolescent-focused therapy (AFT). AFT aims at identifying and scrutinizing eating disorder symptoms in the context of developmental challenges, whereas in FBT, parents' skills are strengthened to promote their child's weight gain and the normalization of eating disorder symptoms. In the well known study by Lock et al. [14], which compared AFT with FBT, significantly more patients in the FBT group achieved remission at the 6-month and 12-month follow-ups. However, during the subsequent 4-year period, more patients in the AFT group had gained additional weight and had recovered from anorexia nervosa than those in the FBT group. Thus, although FBT works more quickly, AFT does not seem to be less effective in the long run [28]. Only $30 \%$ of the FBT group remained weight-restored at the 4-year follow-up (note, however, the high drop-out rate of more than one-third).

In their review, Blessit et al. [29] emphasized the effectiveness of family therapy for the treatment of adolescent anorexia nervosa but claimed that more studies comparing family therapy with other treatment strategies are needed. In a very recent study of 107 adolescents with anorexia nervosa, FBT was compared with parent-focused treatment (PFT). In PFT, the therapist meets with the parents only, whereas the patient is cared for by an experienced nurse. The intervention was conducted in 18 outpatient sessions over a period of 6 months. At the end of treatment, significantly more patients in the PFT arm had achieved remission, defined by an ageappropriate BMI and the normalization of eating disorder psychopathology; at the 6-month and 12month follow-ups, this difference was no longer statistically significant [30""]. Another important multicentre trial compared FBT with systemic family therapy in 164 12-18-year-old patients. Systemic therapy focuses on the family process itself. At the end of treatment and at the 1-year follow-up, remission rates, defined by a restoration of body weight, were similar in the two treatment arms. However, patients in the FBT arm spent fewer days in the hospital, produced lower treatment costs and gained weight faster at the beginning of treatment. Furthermore, patients with more severe obsessive- 
compulsive symptoms benefitted more from systemic treatment [31].

Eisler et al. [32"] conducted the first RCT of Multifamily Therapy (MFT) for adolescent anorexia nervosa and compared it with single family FBT. Patients were significantly improved, defined as achieving a good or intermediate outcome according to the Morgan and Russell scales, in both treatment arms, with $60 \%$ in the single FBT and $75 \%$ in the multifamily arm. At the 18-month follow-up, the difference between the treatment arms was no longer significant, with 57 and 78\% having a good or intermediate Morgan and Russell score in the FBT and the multifamily group, respectively. However, more than one-quarter of the participants had dropped out of the study.

There is emerging evidence that early response to treatment, for example early weight gain in anorexia nervosa, predicts symptom remission in anorexia nervosa (meta-analysis [33",34]), which seems to be independent of different treatment interventions (outpatient or inpatient treatment, FBT, AFT, CBT-E).

Recent reports emphasize the importance of family involvement and 'caring for the caregivers'. Family members of patients with anorexia nervosa, especially those with a chronic course, experience high levels of distress and burden and often suffer from mental disorders themselves [35]. The group of Treasure and Schmidt in London developed a skills training intervention programme comprising a book, DVDs and five telephone calls for caregivers [experienced caregivers helping others (ECHO)] and conducted a pragmatic RCT. The sample comprised 178 individuals treated previously as inpatients who were randomized to either the active arm with the caregiver programme in addition to TAU or to TAU only; of the participants, 11 were adolescents. Patients in the ECHO group showed significantly reduced bed use 7-12 months after discharge [36]. At the 2-year follow-up, there were small to moderate improvements in all patient and caregiver variables; however, they failed to reach significance $\left[37^{\prime \prime}\right]$. The intervention was likely too short to maintain the amelioration.

\section{BULIMIA NERVOSA}

There is some agreement that most adolescents with bulimia nervosa should be treated on an outpatient basis. The medical, psychiatric and psychosocial indications for hospital admission are similar to those for anorexia nervosa. Especially electrolyte disturbances, for example hypokalaemia, suicidal behaviour and severe self-harm, call for inpatient treatment. Apart from psychotherapy, the primary need is for regular meals and snacks to be eaten throughout the day to prevent hunger and craving for food. The meal plan is based on caloric needs and balanced food components. Apart from the binges, patients with bulimia nervosa tend to eat only 'healthy' and low-caloric food. In treatment, they should be encouraged to include so-called forbidden food to prevent craving for sweets or other highcalorie substances.

Similar to the progress made with anorexia nervosa, some recent progress has been made in psychological approaches to bulimia nervosa. In one study, CBT-E was applied to adolescents aged 13-19 years who were not underweight; approximately onethird had bulimia nervosa, 20\% suffered from BED and the remaining half suffered from other eating disorders not specified. According to the authors, two-thirds responded to treatment, showing a marked reduction in eating disorder psychopathology. However, there was no control condition [38].

To my knowledge, two recent methodologically sound RCTs have been conducted. In the two-centre trial by Le Grange et al. [39"'], 130 adolescents with threshold and subthreshold bulimia nervosa were randomly assigned to two specific interventions, FBT-bulimia nervosa and CBT-A (CBT adapted for adolescents). A smaller proportion of patients was assigned to supportive psychotherapy; however, the latter was not sufficiently powered for a statistical analysis and was not included in the evaluation. At discharge and the 6-month follow-up, significantly more patients in the FBT group abstained from binging and purging behaviour; at the 12-month follow-up, a significant difference between the two arms was no longer detectable. However, the results were biased by a drop-out rate of more than one-third at the 6-month and 12-month follow-ups.

The second RCT compared CBT and psychodynamic psychotherapy [40"'] in a sample of 81 female adolescents and young adults with a mean age of 18.7 years. The manualized psychodynamic approach was specifically designed for young people with bulimia nervosa [41], whereas the CBT manual was mainly based on Fairburn's [25] instructions. Remission was defined as the absence of a diagnosis of bulimia nervosa or partial bulimia nervosa according to Diagnostic and Statistical Manual of Mental Disorders, 4th edition (DSM-IV). Patients received a mean of approximately 37 sessions, which is higher than that in most studies on adolescent CBT and FBT. The high drop-out rate was $30 \%$ in this study.

In both RCTs remission rates - albeit defined a little differently - were similar and occurred in approximately one-third of the patients. 
AVOIDANT/RESTRICTIVE FOOD INTAKE DISORDER

ARFID was introduced in the DSM-5 to describe children who are characterized by one or more of the following symptoms: significant weight loss, significant malnourishment, dependence on enteral feeding or additional nutritional supplements and impaired psychosocial functioning. ARFID most commonly develops in infancy or early childhood; clinicians sometimes find it difficult to distinguish this eating disorder from childhood anorexia nervosa. According to my own and other authors experience, the first point of contact is typically a general paediatrician or family doctor [42]. However, patients with ARFID do not present with symptoms of body image disturbance and do not refuse weight gain. In the majority of patients, age of onset is earlier than that for anorexia nervosa, and there is a higher proportion of boys, although ARFID may develop into anorexia nervosa in a minority of cases [23].

In a significant portion of patients, ARFID is associated with distressing medical conditions, for example neurological or gastroenterological problems, or with neurodevelopmental disorders, such as autism and/or ADHD. Accordingly, this disorder may be relatively often observed in preterm infants who do not manage the change from nastrogastric to autonomous oral feeding. In other patients diagnosed with this disorder, important psychiatric problems, such as anxiety, obsessive-compulsive or somatoform disorders, for example emetophobia, food neophobia, choking phobia or phobias of diarrhoea or encopresis, are evident. If symptoms of ARFID are associated with parental neglect or abuse, they will typically vanish with a change in caregivers.

On the contrary, there is a dearth of treatment studies, and there are no evidence-based recommendations. In a recent meta-analysis of 11 studies comprising nearly 600 patients fulfilling diagnostic criteria of ARFID with complex medical and/or neurodevelopmental problems, $70 \%$ of patients at discharge and $80 \%$ at follow-up could be weaned from tube feeding. Interventions were mostly performed by a multidisciplinary team, including nutritional therapists, paediatricians, psychologists and speech and occupational therapists. Treatment approaches were based on behavioural interventions (escape extinction; reinforcement) and withdrawal of tube feeding ('provocation of hunger') and always involved the caregivers [43"']. Other studies indicate a similar intervention strategy; however, there are no further systematic investigations or RCTs $[23,44]$. Very little data exist on the use of medication. In a recent study comprising 127 children between 7 and 80 months with feeding difficulties but not exclusively fulfilling the DSM-5 criteria for ARFID, cyproheptadine, an antihistaminergic and antiserotonergic agent, was applied. Tube-fed patients were excluded. Medication was embedded in a complex multidisciplinary treatment approach. The authors reported significant weight gain and a positive change in mealtime and feeding behaviours [45]. Side effects such as drowsiness, irritability and constipation were reported in $14 \%$ of the children but were mild and often wore off after a few days.

\section{CONCLUSION}

Substantial progress has been made in conducting large and methodologically sound systematic reviews and RCTs of specialized treatment of adolescent anorexia nervosa and, to a lesser extent, of bulimia nervosa. We have learned to optimize weight restoration in malnourished adolescents and to avoid refeeding complications; however, we still do not know the optimal target weight to support long-term remission. Psychotherapeutic research is dominated by FBT mostly performed by the same working group comparing different variants of this treatment strategy. There is an urgent need for a replication of the results and for sufficiently powered trials to ascertain the superiority of FBT over individual treatment, especially in the long-term (Zeeck et al., unpublished data). Regardless of the intervention, only approximately one-third to one-half of patients remains weightrestored in the long run, and these numbers might still be overestimated because of substantial dropout rates at follow-up. Thus, there is an urgent need for studies on preventive measures to permanently maintain remission and on how to help patients with primary treatment-resistant anorexia nervosa. Regarding ARFID, we are only at the beginning; evidence-based treatment guidelines must be developed to support therapists, parents and patients in coping with this often debilitating and stressful disorder.

\section{Acknowledgements}

B.H.-D. is currently receiving grants from the German Ministry of Education and Research, from the Ministry of Health of North-Rhine-Westphalia and from the Technical University of Aachen.

\section{Financial support and sponsorship}

None.

\section{Conflicts of interest}

There are no conflicts of interest. 


\section{REFERENCES AND RECOMMENDED}

\section{READINC}

Papers of particular interest, published within the annual period of review, have been highlighted as:

of special interest

- of outstanding interest

1. Murray CJL, Barber RM, Foreman $\mathrm{KJ}$, et al. Global, regional, and national disability-adjusted life years (DALYs) for 306 diseases and injuries and healthy life expectancy (HALE) for 188 countries, 1990-2013: quantifying the epidemiological transition. Lancet 2015; 386:2145-2191.

2. Erskine HE, Whiteford HA, Pike KM. The global burden of eating disorders.

- Curr Opin Psychiatry 2016; 29:346-353.

The authors describe the inclusion of anorexia and bulimia nervosa in the Global

Burden of Disease Study 2013 as a noteworthy event in the recognition of the

importance of eating disorders in the global health community.

3. Hoek HW. Review of the worldwide epidemiology of eating disorders. Curr Opin Psychiatry 2016; 29:336-339.

4. Steinhausen $\mathrm{H}-\mathrm{C}$, Jensen $\mathrm{CM}$. Time trends in lifetime incidence rates of firsttime diagnosed anorexia nervosa and bulimia nervosa across 16 years in a Danish nationwide psychiatric registry study. Int J Eat Disord 2015; 48:845850.

5. Keski-Rahkonen A, Mustelin L. Epidemiology of eating disorders in Europe:

- prevalence, incidence, comorbidity, course, consequences, and risk factors. Curr Opin Psychiatry 2016; 29:340-345.

Comprehensive review on European epidemiologic data of eating disorders.

6. Hoang U, Goldacre M, James A. Mortality following hospital discharge with a diagnosis of eating disorder: national record linkage study, England, 2001 2009: mortality following a diagnosis of eating disorder. Int J Eat Disord 2014; 47:507-515.

7. Brown A, McClelland J, Boysen E, et al. The FREED Project (first episode and

- rapid early intervention in eating disorders): service model, feasibility and acceptability: early intervention in eating disorders. Early Interv Psychiatry 2016. [Epub ahead of print]

First description of an important model for early intervention in eating disorders.

8. Lock J, La Via MC; American Academy of Child and Adolescent Psychiatry (AACAP) Committee on Quality Issues (COI). Practice parameter for the assessment and treatment of children and adolescents with eating disorders. J Am Acad Child Adolesc Psychiatry 2015; 54:412-425.

9. Hay $P$, Chinn D, Forbes D, et al. Royal Australian and New Zealand College of Psychiatrists clinical practice guidelines for the treatment of eating disorders. Aust N Z J Psychiatry 2014; 48:977-1008.

10. Madden S, Miskovic-Wheatley J, Wallis A, et al. A randomized controlled trial of in-patient treatment for anorexia nervosa in medically unstable adolescents. Psychol Med 2015; 45:415-427.

11. Herpertz-Dahlmann $B$, Schwarte $R$, Krei $M$, et al. Day-patient treatment after

- short inpatient care versus continued inpatient treatment in adolescents with anorexia nervosa (ANDI): a multicentre, randomised, open-label, noninferiority trial. Lancet 2014; 383:1222-1229.

Large randomized controlled trial (RCT) to compare inpatient and day patient treatment in adolescent anorexia nervosa.

12. Madden S. Systematic review of evidence for different treatment settings in anorexia nervosa. World J Psychiatry 2015; 5:147.

13. Friedman $\mathrm{K}$, Ramirez $\mathrm{AL}$, Murray $\mathrm{SB}$, et al. $\mathrm{A}$ narrative review of outcome

- studies for residential and partial hospital-based treatment of eating disorders. Eur Eat Disord Rev 2016; 24:263-276.

Thorough overview of observation studies and RCTs on the effect of different treatment settings on outcome.

14. Lock J, Le Grange D, Agras WS, et al. Randomized clinical trial comparing family-based treatment with adolescent-focused individual therapy for adolescents with anorexia nervosa. Arch Gen Psychiatry 2010; 67:1025.

15. Herpertz-Dahlmann B, van Elburg A, Castro-Fornieles J, Schmidt U. ESCAP Expert Paper: new developments in the diagnosis and treatment of adolescent anorexia nervosa - a European perspective. Eur Child Adolesc Psychiatry $2015 ; 24: 1153-1167$.

16. Dempfle A, Herpertz-Dahlmann B, Timmesfeld N, et al. Predictors of the resumption of menses in adolescent anorexia nervosa. BMC Psychiatry 2013; 13:308.

17. Faust JP, Goldschmidt AB, Anderson KE, et al. Resumption of menses in anorexia nervosa during a course of family-based treatment. J Eat Disord 2013; $1: 12$.

18. Rocks $T$, Pelly F, Wilkinson $P$. Nutrition therapy during initiation of refeeding in underweight children and adolescent inpatients with anorexia nervosa: a systematic review of the evidence. J Acad Nutr Diet 2014; 114:897-907.

19. Maginot TR, Kumar MM, Shiels J, et al. Outcomes of an inpatient refeeding protocol in youth with anorexia nervosa: Rady Children's Hospital San Diego/ University of California, San Diego. J Eat Disord 2017; 5:1.

20. Garber AK, Sawyer SM, Golden NH, et al. A systematic review of approaches to refeeding in patients with anorexia nervosa. Int J Eat Disord 2016; 49:293310.

Profound systematic review on 27 studies in anorexia nervosa investigating different renutrition approaches and their association with the medically serious refeeding syndrome leading to eight important conclusions for clinicians.
21. O'Connor G, Nicholls D, Hudson L, Singhal A. Refeeding low weight

n. hospitalized adolescents with anorexia nervosa: a multicenter randomized controlled trial. Nutr Clin Pract 2016; 31:681-689.

First relevant, although small RCT on the comparison of a lower caloric supply vs. higher caloric supply at the beginning of refeeding in adolescent anorexia nervosa. The latter diet was associated with better weight gain but not with a higher rate of medical problems, including hypophosphatemia. The trial will contribute to a change in refeeding approaches in clinical practise.

22. Herpertz-Dahlmann $B$, Salbach-Andrae $H$. Overview of treatment modalities in adolescent anorexia nervosa. Child Adolesc Psychiatr Clin N Am 2009; 18:131-145.

23. Mairs R, Nicholls $D$. Assessment and treatment of eating disorders in children and adolescents. Arch Dis Child 2016; 101:1168-1175.

24. Murphy R, Straebler S, Cooper Z, Fairburn CG. Cognitive behavioral therapy for eating disorders. Psychiatr Clin North Am 2010; 33:611-627.

25. Fairburn CG. Cognitive behavior therapy and eating disorders. New York: Guilford Press; 2008; ISBN 978-1593857097.

26. Dalle Grave R, Calugi S, Doll HA, Fairburn CG. Enhanced cognitive behaviour therapy for adolescents with anorexia nervosa: an alternative to family therapy? Behav Res Ther 2013; 51:R9-R12.

27. Dalle Grave R, Calugi S, El Ghoch $M$, et al. Inpatient cognitive behavior therapy for adolescents with anorexia nervosa: immediate and longer-term effects. Front Psychiatry 2014; 5:14.

28. Le Grange $D$, Lock J, Accurso EC, et al. Relapse from remission at two- to four-year follow-up in two treatments for adolescent anorexia nervosa. J Am Acad Child Adolesc Psychiatry 2014; 53:1162-1167.

29. Blessitt E, Voulgari S, Eisler I. Family therapy for adolescent anorexia nervosa. Curr Opin Psychiatry 2015; 28:455-460.

30. Le Grange D, Hughes EK, Court A, et al. Randomized clinical trial of parent-

-1 focused treatment and family-based treatment for adolescent anorexia nervosa. J Am Acad Child Adolesc Psychiatry 2016; 55:683-692.

This significant study demonstrated that conjoint family sessions are no more effective than separate sessions for parents and patients and thus questioned a long-standing paradigm. Moreover, it confirmed the everyday experience of many clinicians.

31. Agras WS, Lock J, Brandt H. Comparison of 2 family therapies for adolescent anorexia nervosa: a randomized parallel trial. JAMA Psychiatry 2014; $71: 1279-1286$.

32. Eisler I, Simic M, Hodsoll J, et al. A pragmatic randomised multicentre trial of

multifamily and single family therapy for adolescent anorexia nervosa. BMC Psychiatry 2016; 16:422.

Large RCT demonstrating the effect of MFT on treatment outcome in comparison with the more traditional single family-based therapy (FBT). However, families randomized to the MFT-anorexia nervosa group were all initially engaged in treatment individually so that the 'overall' effect might be biased by primarily single family therapy.

33. Nazar BP, Gregor LK, Albano G. Early response to treatment in eating

- disorders: a systematic review and a diagnostic test accuracy meta-analysis. Eur Eat Disord Rev 2017; 25:67-79.

Interesting systematic review of the prognostic positive value of early symptom improvement in treatment of eating disorders.

34. Madden S, Miskovic-Wheatley J, Wallis A, et al. Early weight gain in familybased treatment predicts greater weight gain and remission at the end of treatment and remission at 12-month follow-up in adolescent anorexia nervosa. Int J Eat Disord 2015; 48:919-922.

35. Schwarte R, Timmesfeld N, Dempfle A, et al. Expressed emotions and depressive symptoms in caregivers of adolescents with first-onset anorexia nervosa-a long-term investigation over 2.5 years. Eur Eat Disord Rev 2017; 25:44-451

36. Hibbs R, Magill N, Goddard E, et al. Clinical effectiveness of a skills training intervention for caregivers in improving patient and caregiver health following in-patient treatment for severe anorexia nervosa: pragmatic randomised controlled trial. BJPsych Open 2015; 20:56-66.

37. Magill N, Rhind $\mathrm{C}$, Hibbs $\mathrm{R}$, et al. Two-year follow-up of a pragmatic

- randomised controlled trial examining the effect of adding a carer's skill training intervention in inpatients with anorexia nervosa. Eur Eat Disord Rev 2016; 24:122-130.

In this pragmatic randomized trial, the effects of a carer's skills programme added to treated under usual was measured as patient, caregiver and service outcome. The small/moderate size improvements in patients' and caregivers' outcome variables were sustained over the observation period but failed to reach significance at the 2-year follow-up. The results emphasize the importance of providing support to carers after their child's discharge from hospital, however suggest a longer duration of the assistance.

38. Dalle Grave R, Calugi S, Sartirana M, Fairburn CG. Transdiagnostic cognitive behaviour therapy for adolescents with an eating disorder who are not underweight. Behav Res Ther 2015; 73:79-82.

39. Le Grange D, Lock J, Agras WS, et al. Randomized clinical trial of family-based

- treatment and cognitive-behavioral therapy for adolescent bulimia nervosa. J Am Acad Child Adolesc Psychiatry 2015; 54:886-894.

In this large outpatient RCT, CBT and FBT were compared in adolescents. In the FBT-group, patients achieved higher abstinence rates in a shorter period of time; however, at 1-year follow-up, remission rates did no longer differ. To our knowledge, this is the first RCT to compare FBT and individually based therapy in adolescent bulimia nervosa and an important contribution to our knowledge for treatment of bulimia nervosa. 
40. Stefini A, Salzer S, Reich G, et al. Cognitive-behavioral and psychodynamic -1. therapy in female adolescents with bulimia nervosa: a randomized controlled trial. J Am Acad Child Adolesc Psychiatry 2017; 56:329-335.

This RCT looked at differences in outcome between cognitive-behavioural and psychodynamic therapy, the latter without a specific focus on eating disorder symptoms in female adolescents. Although the theoretical framework and practical implementation differed essentially, there was no statistical difference in primary (rate of remission) or secondary outcome measures. This raises the question about some broader effect of psychotherapeutic approaches outside the effect on eating disorder psychopathology.

41. Reich $\mathrm{G}$, Horn $\mathrm{H}$, Winkelmann $\mathrm{K}$, et al. Psychodynamic focal therapy of bulimia nervosa for female adolescents and young adults. Prax Kinderpsychol Kinderpsychiatr 2014; 63:2-20.

42. Norris ML, Spettigue WJ, Katzman DK. Update on eating disorders: current perspectives on avoidant/restrictive food intake disorder in children and youth. Neuropsychiatr Dis Treat 2016; 12:213-218.
43. Sharp WG, Volkert VM, Scahill L, et al. A systematic review and meta-analysis -1. of intensive multidisciplinary intervention for pediatric feeding disorders: how standard is the standard of care? J Pediatr $2017 ; 181: 116-124 \mathrm{e} 4$

This systematic review involving about 600 patients is one of the first to analyze treatment outcome in children with chronic food refusal. All patients included fulfilled DSM-5 criteria for avoidant/restrictive food intake disorder and the majority also had complex medical and/or developmental histories. The results indicate a necessity for a multidisciplinary team approach applying behavioral interventions and gradual tube weaning. Moreover, the review highlights the necessity for evaluation and standardization of interventions for this serious disorder and the development of clinical guidelines.

44. Cardona Cano S, Hoek HW, Bryant-Waugh R. Picky eating: the current state of research. Curr Opin Psychiatry 2015; 28:448-454.

45. Sant'Anna AMGA, Hammes PS, Porporino M, et al. Use of cyproheptadine in young children with feeding difficulties and poor growth in a pediatric feeding program. J Pediatr Gastroenterol Nutr 2014; 59:674-678. 